\title{
Study of Likelihood of Infection with COVID 19 Based on Source of Exposure to Infection among Hospital Staff
}

\author{
Jayalakshmi T.K. ${ }^{1}$, Bhumika Madhav², Narendra Patil ${ }^{3}$, Dipti Dhanwate ${ }^{4}$, \\ Prakash Cherath ${ }^{5}$, Dhanaji Revande ${ }^{6}$,Jay Shinde ${ }^{7}$, Monhiki Pasweti ${ }^{8}$ \\ 1, 2, 6 Department of Pulmonary Medicine, Apollo Hospitals, Navi Mumbai, Maharashtra, India. \\ 3, 4 Department of Microbiology, Apollo Hospitals, Navi Mumbai, Maharashtra, India. \\ ${ }^{5}$ Department of Statistics, VESIM, Mumbai, Maharashtra, India. ${ }^{7} \mathrm{KEM}$ Hospital, Mumbai, \\ Maharashtra, India. ${ }^{8}$ Apollo Hospitals, Navi Mumbai, Maharashtra, India.
}

\section{ABSTRACT}

\section{BACKGROUND}

The corona virus disease - 19 (COVID - 19) pandemic has caused significant morbidity and mortality throughout the world, as well as major social, educational and economic disruptions. Hospital staff have maximum exposure making it extremely crucial to find a solution to reduce the disease burden among the hospital staff. A sample of 170 consecutive hospital staff infected with COVID in the months from May 2020 to December 2020 was studied. It was a retrospective study done by analysis of out-patient department (OPD) and indoor patient records in that time period.The purpose of this study was to assess different sources and types of exposure of hospital staff to COVID 19 infection and prevent episodes of infection in hospital staff.

\section{METHODS}

Survey of 170 COVID positive staff at a tertiary hospital was conducted and their potential sources of infection were documented. Source of infection could be other staff in hospital, room mates, patients, patient relatives, family, personal protective equipment (PPE) donning and doffing errors. Types of interaction could be in covid or non-covid areas of the hospital or during clinical encounters or social encounters within the hospital.

\section{RESULTS}

Nurses (46\%) and doctors (29\%) constitute $75 \%$ of the total heath care staff infections in the hospital. Desk job staff, attendants, food and beverage workers and drivers together constitute $25 \%$ of the total health care staff infections in the hospital. The most common sources of infection were found to be social interaction and patient interaction. The infections were acquired much more in the non-covid areas of the hospital than in the covid areas. $65 \%$ of infections were acquired within three days of exposure and $87 \%$ of total infections were acquired within 6 days of exposure. $77 \%$ of infections were acquired within the hospital premises in patient and social interaction cases.

\section{CONCLUSIONS}

Staff acquisition of COVID was found to be significantly higher in non-covid than in covid areas. Social distancing measures, mask use, hand hygiene, attention to PPE would help in significant drop in new COVID infections in hospital.
Corresponding Author: Dr.Bhumika Madhav, \#43/1, RCF Row Houses, Sector 6, Vashi, Navi Mumbai-400703, Maharashtra, India. E-mail: bhumikamadhav@yahoo.com

DOI: $10.14260 / j e m d s / 2021 / 778$

How to Cite This Article:

Jayalakshmi TK, Madhav B, Patil N, et al. Study of likelihood of infection with COVID 19 based on source of exposure to infection among hospital staff. J Evolution Med Dent Sci 2021;10(45):3849-3852, DOI: $10.14260 /$ jemds $/ 2021 / 778$

Submission 05-11-2021, Peer Review 06-12-2021, Acceptance 13-12-2021, Published 21-12-2021.

Copyright (c) 2021 JEMDS. This is an open access article distributed under Creative Commons Attribution License [Attribution 4.0 International (CC BY 4.0)]

\section{KEY WORDS}

COVID 19, Infection, Health Care Workers, Pandemic Prevention. 


\section{BACKGROUND}

In December 2019, a novel coronavirus was identified, believed to have originated from Wuhan, China. It was more contagious than previous severe acute respiratory syndrome (SARS) or Middle east respiratory syndrome (MERS) coronavirus. Initial focus was on China, and later shifted to Europe, North America and then to the entire world.

The World Health Organization (WHO) declared the COVID-19 as a pandemic on 11 th March 2020. Governments all over the world closed their borders and imposed lockdown to curtail its spread. Since then, there have been new strains and variants, each more contagious and deadly than the last.

The COVID-19 pandemic has caused morbidity and mortality throughout the world, along with major social, educational and economic disruptions. The first case in India was reported on $30^{\text {th }}$ January 2020 in the state of Kerala. Cases peaked in September 2020 with India having the largest number of confirmed cases in Asia. The number of cases per million population and deaths per million population were significantly lower than the countries like USA, Italy, Brazil, France, Russia. ${ }^{1}$

Healthcare services in the country were stretched thin and hospitals struggled to keep staff and patients safe. There was an increase in the incidence of hospital acquired infections, ${ }^{2,3}$ under staffing and lack of resources.

Safety of healthcare workers needs to be ensured to prevent the transmission of the disease and also to protect the staff as well as their family members.

It is extremely crucial to understand behaviours of hospital staff, including the wearing of appropriate personal protective equipment which is important for prevention of COVID 19 illness. It is also necessary to educate the staff at regular intervals regarding the possible sources of infection such as social interaction, improper use of masks, poor hand hygiene, proper use of PPE kits etc.

Signs and symptoms of COVID 19 illness most commonly include fever, cough, shortness of breath. A few patients can develop pneumonia or severe acute respiratory syndrome and death. The other symptoms of COVID 19 illness include diarrhoea, loss of smell, loss of taste, conjunctivitis, rash, malaise etc. As per the WHO recommendation, in order to prevent the spread of COVID-19 infection, standard recommendation includes frequent cleaning of hands using alcohol-based hand rub or soap and water; covering the nose and mouth with a elbow or disposable tissue when coughing and sneezing; and avoiding close contact with anyone that has a fever and cough. It is essential to wear masks at all times when in contact with a patient or colleague. It is recommended to use an N95 mask when treating a COVID 19 patients or a suspect patient and at least use a surgical 3 ply mask when dealing with non suspect patients. Adequate measures of social distancing need to be in place at all times. In order to prevent the spread of this outbreak, WHO is working with global experts, governments and partners to rapidly expand scientific knowledge on this virus.

The current study aims to look at the different sources and types of exposure of hospital staff to COVID 19 infection, identify likely sources and prevent episodes of infection by making protocols and educating the staff from time to time.

\section{METHODS}

A retrospective observational study with the study subjects being all staff who were COVID-19 positive admitted at a large tertiary hospital from May 2020 to December 2020 was conducted. Survey of 170 covid positive staff at a tertiary hospital was conducted and their potential sources of infection were documented.The age of patients was from 20 to 60 years. Vaccination was not available at the time of study.. The study population was $100 \%$ of hospital staff who were COVID positive. RT - PCR, rapid antigen test (RAT) and cartridge based nucleic acid amplification test (CBNAAT) were the tests used for confirming positive diagnosis. Source of infection could be other staff in hospital, room mates, patients, patient relatives, family, PPE donning and doffing errors. Types of interaction could be in covid or non-covid areas of the hospital or during clinical encounters or social encounters within the hospital. Some patients had multiple possible sources.

\section{Statistical Analysis}

The research objective drove the analysis of data. All assumptions were tested in hypothesis testing. Data was analyzed using descriptive and inferential statistics.

\section{RESULTS}

After analysis of collected data, the following results were obtained. There were 88 (52\%) female and 82 (48\%) male patients.

In our study, $78 \%$ of the patients belonged to the age group of 20 to 35 years and $22 \%$ of the patients belonged to the age group of 36 to 54 years.

Of all the staff that were infected with COVID 19 illness, $29 \%$ were doctors, $46 \%$ were nurses, $18 \%$ were staff with desk Job such as receptionist and guest relationship manager etc. $5 \%$ of all staff that were infected included attendants. 2 $\%$ were drivers and $1 \%$ of the staff belonged to food and beverage. (Figure 1)

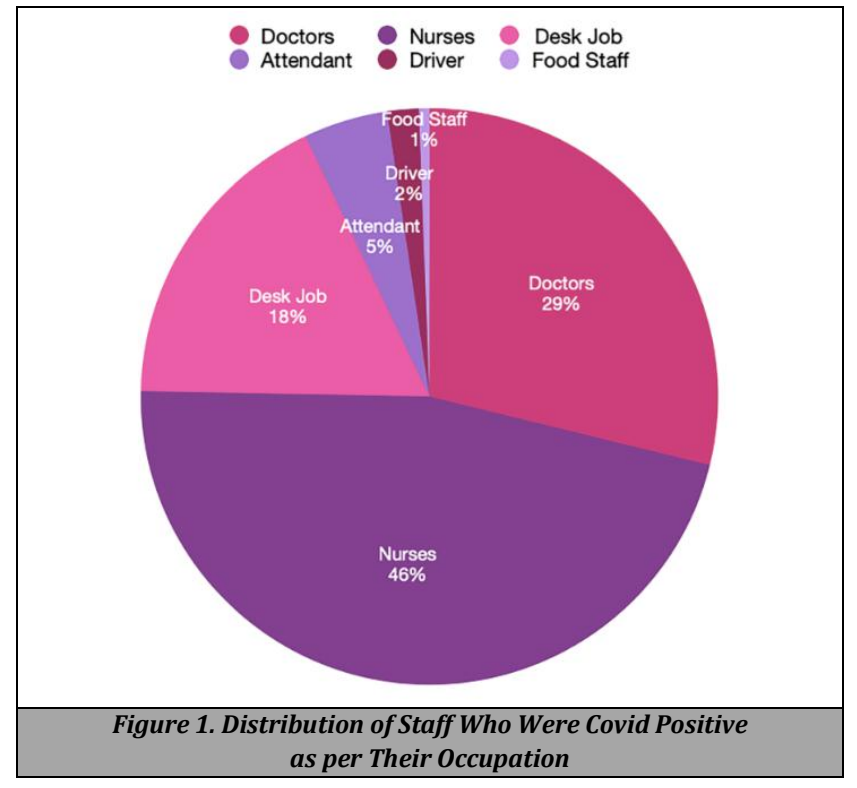


Source of infection could have been through roommates, patient, patient's relatives, family, social interaction and donning and doffing errors. We found in our study that maximum source of infection was through direct patient interaction followed by social interaction with other staff in the hospital, example while having food or tea together or travelling together to work. (Figure 2)
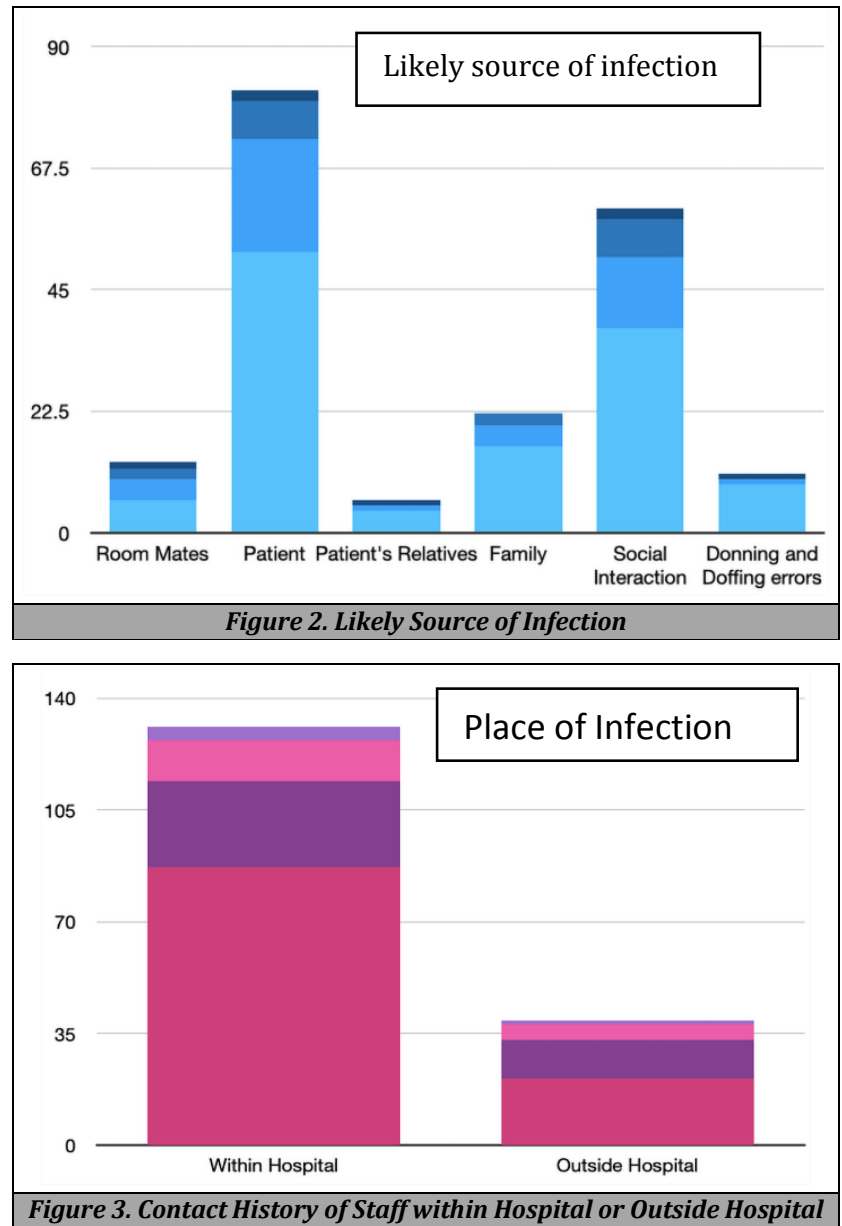

Maximum staff got the infection while working in the hospital and (Fig. 3)

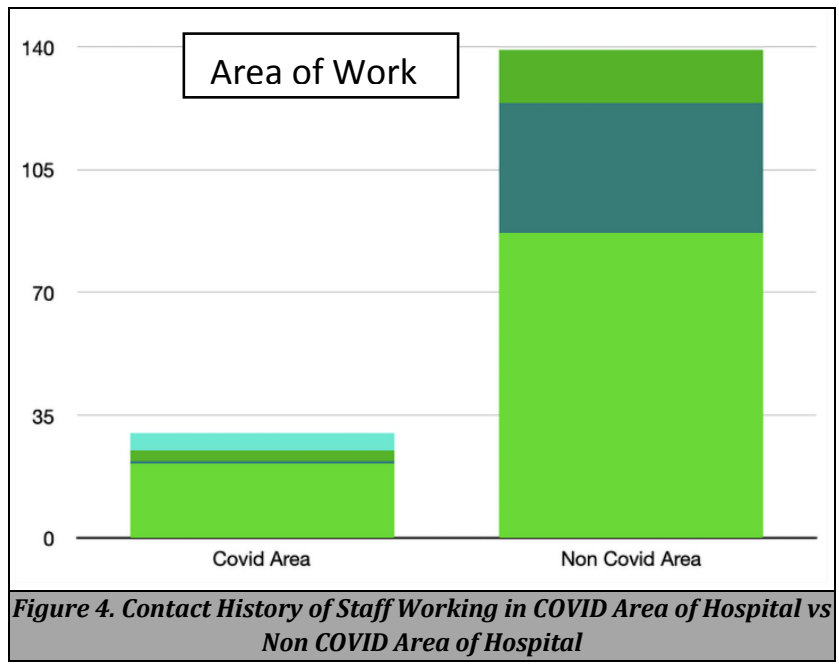

Most of these staff were working in the non covid area. 25 out of 170 staff were working in the COVID area and 145 were working in the non covid area. (Fig. 4).

\section{DISCUSSION}

COVID-19 is spread by droplets and particles from an infected individual. These can directly infect another person or contaminate surfaces that can transmit the disease by direct contact. Airborne transmission occurs in aerosol generating procedures and remain suspended for long in air over long distances and time. ${ }^{4}$ Mode of entry is through the eyes, nose and mouth. ${ }^{5}$

This study showcases the different sources of infection for hospital staff. The number of infected males and females were almost equal. Greater numbers were found in younger age group of 20 - 35 years. Nursing was the occupation group with the highest number of infections.

The most common sources of infection were found to be patient interaction and social interaction. The infections were acquired much more in the hospital than outside, while noncovid areas of the hospital had higher number than the covid areas. $65 \%$ of infections were acquired within three days of exposure and $87 \%$ of total infections were acquired within 6 days of exposure. $77 \%$ of infections were acquired within the hospital premises in patient and social interaction cases.

A similar study of 274 healthcare workers conducted by Banerjee, Abhra et al. also showed increased infection rates in non-frontline workers (56 \%) as compared to frontline workers (44\%) and higher number of infections in age group of below 45 years. ${ }^{6}$

Using phylogenetic analysis, Sikkens, Jonne et al. noted an increase risk of HCW to HCW transmission in regular COVID wards, but rates of intensive care units (ICUs) and emergency departments (EDs) were similar to non-covid areas. ${ }^{7}$

The findings of an epidemiological study by Chou, Roger et al. which uses data from over 100 different studies states that there is no consistent association between risk and age, sex or healthcare worker role (doctor vs nurses). Risk of infection showed negative association with appropriate PPE and mask use, while direct exposure and aerosol generation procedures have a positive correlation. ${ }^{8}$

Aerosol generating procedures and certain specialities like anesthesiology, otolaryngology, dentistry have a greater exposure ${ }^{9}$ but have a decreased risk of infection due to more use of appropriate PPE and ventilated procedure rooms. There is increased occurrence of aerosol generating events like coughing and sneezing in non procedure areas.

These results bring to light the need for preventive measures and current lack of them in non-covid areas and social settings.

Apart from standard practices to stop the spread of disease, recommended routine infection control measures for all patients include telehealth, triage, screening, monitoring and re-evaluating, controlling infection at source by using masks and respirators, hand hygiene, physical distancing, universal PPE like n95 masks, respirators, eye protection, targeted testing. Air quality must be optimized to reduce aerosols. Non urgent and elective procedures can be postponed. Response process must be created and guidelines should be followed. 10

All hospital staff needs to be aware of routine disease control measures and practice them in non-healthcare settings as well. Appropriate distancing, educating people about the difference between cloth, surgical three ply and 
n95 masks, steps and moments of hand washing are essential. ${ }^{11}$ Education increases efficacy of hand washing. ${ }^{12}$

\section{CONCLUSIONS}

It is essential that all staff in hospital diligently comply with hand washing, social (physical) distancing, mask wearing and appropriate use of personal protective equipment including strict donning and doffing procedures. These practices should already have become the norm but are often poorly adhered to. Some of the safety practices like proper ventilation, more widespread use of airborne personal protective equipment and formal donning and doffing practices, can be extended to social settings and non covid areas where hospital staff is more at risk.

\section{Limitations of the Study}

The sample size of 170 is small and includes data from workers in only one hospital, therefore cannot be extrapolated to the general population. We were unable to do phylogenetic sequencing analysis as the facility was not available. Multiple possible sources of infection for each patient have been recorded on basis of history.

\author{
Abbreviation's Used \\ CBNAAT- Cartridge Based Nucleic Acid Amplification Test \\ HCW - Health Care Workers \\ ICU - Intensive Care Unit \\ MERS - Middle East Respiratory Disease \\ OPD - Out Patient Department \\ PPE - Personal Protective Equipment \\ RAT - Rapid Antigen Test \\ RTPCR - Reverse Transcription Polymerase Chain Reaction \\ SARS - Severe Acute Respiratory Syndrome \\ WHO -World Health Organization
}

Data sharing statement provided by the authors is available with the full text of this article at jemds.com.

Financial or other competing interests: None.

Disclosure forms provided by the authors are available with the full text of this article at jemds.com.

\section{REFERENCES}

[1] Park K.Park's textbook of preventive and social medicine. $26^{\text {th }}$ edn. India: Banarsidas Bhanot Publishers 2021.

[2] Cook TM, Lennane S. Occupational COVID-19 risk for anaesthesia and intensive care staff - low-risk specialties in a high-risk setting. Anaesthesia 2021;76(3):295-300.

[3] Abo-Leyah H, Gallant S, Cassidy D, et al. The protective effect of SARS-CoV-2 antibodies in Scottish healthcare workers. ERJ Open Res 2021;7(2):00080-2021.

[4] Transmission of SARS-CoV-2: implications for infection prevention precautions [Internet]. Who.int. 2020. Available from: https://www.who.int/newsroom/commentaries/detail/transmission-of-sars-cov-2implications-for-infection-prevention-precautions

[5] Infection Control Guidance for Healthcare Professionals about Coronavirus

(COVID-

19).https://www.cdc.gov/coronavirus/2019ncov/prevent-getting-sick/how-covid-spreads.html

[6] Banerjee A, Mukherjee K, Bhattacharjee D, et al. Status of health-care workers in relation to covid-19 infection: a retrospective study in a level 4 COVIDhospital in Eastern India. [Internet]. Pesquisa.bvsalud.org. 2021 Available from: https://pesquisa.bvsalud.org/global-literature-onnovel-coronavirus-2019-ncov/resource/en/covidwho946701

[7] SikkensJJ, Buis DTP, Peters EJG. Serologic surveillance and phylogenetic analysis of SARS-CoV-2 infection among hospital health care workers. JAMA Network Open 2021;4(7):e2118554.

[8] Bandyopadhyay S, Baticulon RE, Kadhum M. Infection and mortality of healthcare workers worldwide from COVID-19: a systematic review. BMJ Global Health 2020;5(12):e003097.

[9] Interim infection prevention and control recommendations for healthcare personnel during the coronavirus disease.2019 (COVID-19) Pandemic URL https://www.cdc.gov/coronavirus/2019ncov/hcp/infection-control-recommendations.html

[10] World Health Organization. Hand hygiene: why, how \& when? https://www.who.int/gpsc/5may/Hand_Hygiene_Why_ How_and_When_Brochure.pdf

[11] Fries KE, Figueroa AM, Pickerign $H$, et al. Use of World Health Organization guidelines to improve hand washing efficacy. J Contin Educ Nurs 2020;51(10):453-6. 